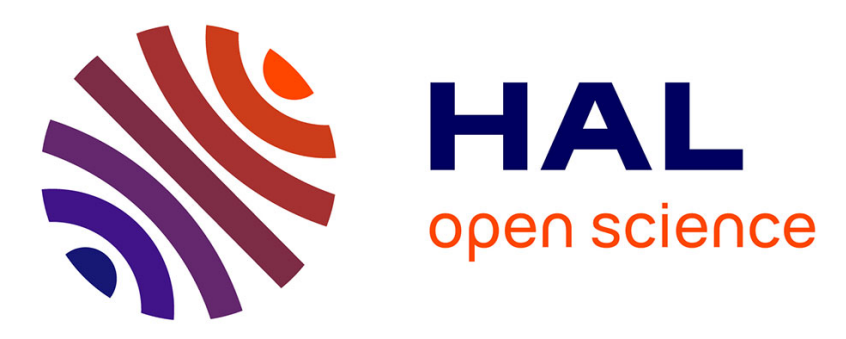

\title{
A Usage Coverage-Based Approach for Assessing Product Family Design
}

Jiliang Wang, Bernard Yannou, Fabrice Alizon, Pierre-Alain Yvars

\section{To cite this version:}

Jiliang Wang, Bernard Yannou, Fabrice Alizon, Pierre-Alain Yvars. A Usage Coverage-Based Approach for Assessing Product Family Design. Engineering with Computers, 2012, pp.DOI 10.1007/s00366-012-0262-1. 10.1007/s00366-012-0262-1 . hal-00748702

\section{HAL Id: hal-00748702 \\ https://hal.science/hal-00748702}

Submitted on 17 Mar 2013

HAL is a multi-disciplinary open access archive for the deposit and dissemination of scientific research documents, whether they are published or not. The documents may come from teaching and research institutions in France or abroad, or from public or private research centers.
L'archive ouverte pluridisciplinaire HAL, est destinée au dépôt et à la diffusion de documents scientifiques de niveau recherche, publiés ou non, émanant des établissements d'enseignement et de recherche français ou étrangers, des laboratoires publics ou privés. 


\title{
A usage coverage based approach for assessing product family design
}

\author{
Jiliang WANG ${ }^{1}$, Bernard YANNOU ${ }^{1}$, Fabrice ALIZON $^{2}$, Pierre-Alain YVARS ${ }^{3}$ \\ 1. Laboratoire Genie Industriel, Ecole Centrale Paris, Grande Voie des Vignes, \\ 92290 Châtenay-Malabry, France \\ 2. Keyplatform, 91 rue du Faubourg St Honore, 75008, Paris, France \\ 3. Laboratoire LISMMA, SUPMECA, 3 rue Fernand Hainaut, 93407 Saint-Ouen \\ Cedex, France
}

Tel: +33 141131521 - Fax: +33 141131272

Email: wangjiliang@gmail.com, bernard.yannou@ecp.fr

\begin{abstract}
Computation techniques have provided designers with deeper understanding of the market niches that were neglected before. Usage contextual information has been studied in marketing research since last century, however little research in design engineering focuses on it. Therefore, in this paper, we analyzed the relations between usage context information and the design of products. A usage coverage model is established to integrate users and their expected usage scenarios into product family assessment. We map the user's individual capacity together with a given product into the usage context space. The overlapping between required usage and feasible usage can be measured. Based on this mechanism, several usage coverage indices are proposed to assess the compliance of a given product family to the expected set of usage scenarios to be covered. The original method is demonstrated on a scale-based product family of jigsaws in a redesign context. Constraint Programming Technique is applied to solve the physics-based causal-loops that determine usage performances in a set-based design approach. Designers can rely on the results to eliminate redundant units in the family or modify the configuration of each product. The contribution of the paper is to provide an inter-disciplinary point of view to assessing the composition and configuration of a product family design.
\end{abstract}

Keywords: Usage model, Usage Coverage Index, Constraint Programming, Product Family Design

\section{Introduction}

"Any customer can have a car painted any color he wants so long as it is black": Henry Ford's famous maxim marks the beginning of meaningful mass production thought in the industrial age. And while Ford's production model was indeed a pioneer in the revolution of mass production, he was also a pioneer in mass customization [1] since more than 5\% of Ford's production was mass customized. Figure 1 shows the evolution of the main production method from 1914 until now: from craft production to mass production, then to lean production and finally to mass customization. Mass customization requires an increasingly distinct number of products on sale while volume per product stays relatively low. This has introduced the challenge of fulfilling various market requirements while keeping production costs low. 


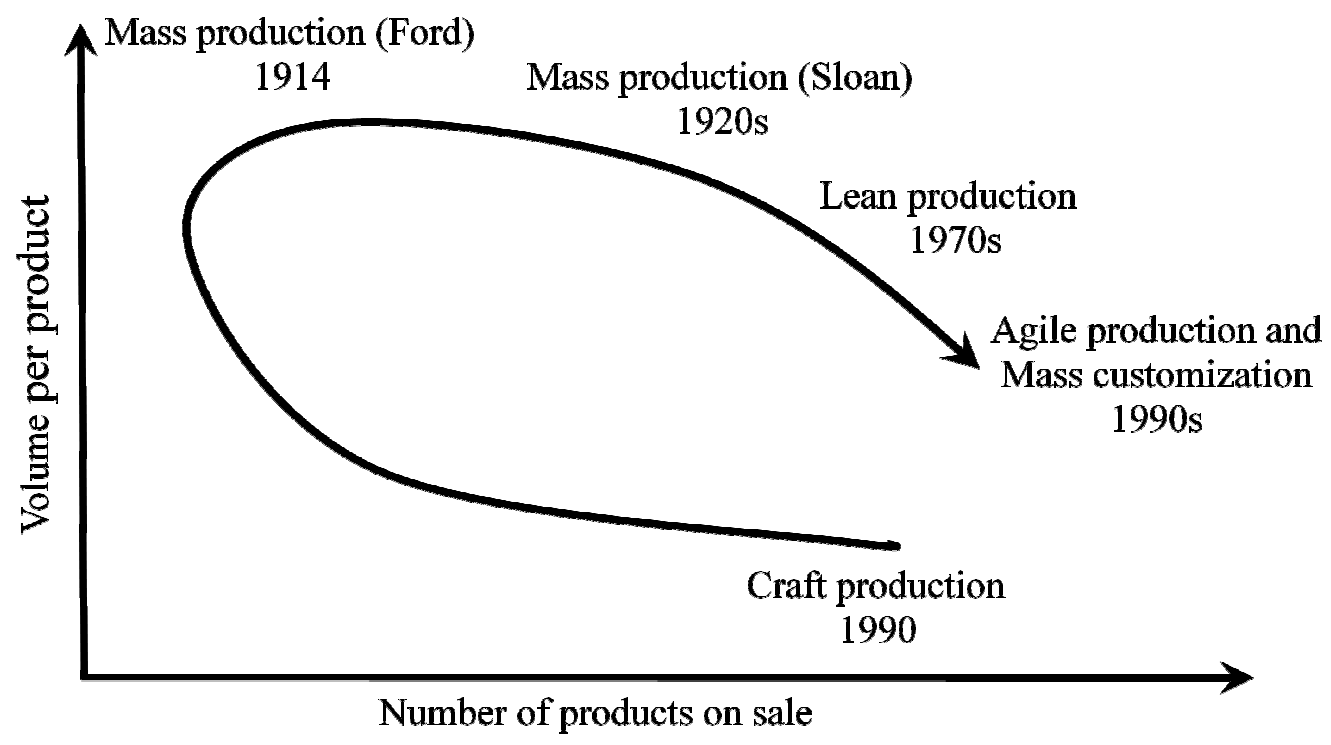

Fig. 1 The evolution of production methods [2]

Many companies are today facing an unprecedented trend of the individualization of demand. The concept of mass customization is defined by Tseng and Jiao [3] as "producing goods and services to meet individual customer's needs with near mass production efficiency". While customization is a well-known strategy in most B-to-B markets, it must be systematically applied to B-to-C markets to better comply with the variety of customer need. A recent Forrester report [4] confirmed the attractiveness to customers of purchasing customized products. Customers are better educated with higher income levels, less likely to compromise, and pay more attention to personal usage adaptation of a given product.

Two issues of design automation in the objective of mass customization are addressed in this paper: introducing more product usage contexts in the market segmentation (see [5]) and conjointly sampling a product family in a more explicit and scientific way.

Indeed, the product family development concept has been widely accepted both in academic and industrial contexts. Companies like Sony, Black \& Decker and Volkswagen, for instance, have successfully implemented strategies to design an entire family of products around modular-based and/or scale-based platforms to satisfy a wide variety of customer requirements, while benefiting from the advantage of economies of scale [6]. The development of product families has made company production processes and supply chains more flexible. The measurement of usage satisfaction for a given product family is a significant factor in the evaluation and decision-making processes of product family design/redesign.

This paper starts by introducing the concept of usage model and usage coverage index, presenting a generic approach to product family assessment from the usage context point of view. During the case study and model resolution phases, domain based variables and inter-variable causal loops are highlighted. To allow a performance calculation in such a case, constraint programming techniques are used to simulate the performances of given products with regard to personalized usage scenarios. New coverage indices are introduced for product family redesign contexts. A case study from an illustrative jigsaw power tool family is presented as a practical example. 


\section{Literature review}

The modeling of usage can be traced to marketing research. Traditional information-processing research in consumer choice behavior has typically contented itself with stimulus and subject task manipulations. Deterministic preference/choice models are usually based on the premise that products are valued for the attributes they possess and that customers seek to maximize their "utility" by choosing desired combinations of attributes [7]. Thus products offering similar combinations of levels of attributes are likely to be more competitive. However, emerging fields of research are seeking to emphasize the role of usage context and usage goals in consumer learning, together with use of that knowledge in decision-making. In the following of the paper we adopt the definition of He et al. [14, 41] for usage context "as all aspects describing the context of product use that vary under different use conditions and affect product performance and/or consumer preferences for the product attributes."

Emphasis on the matching between situational requirements and product benefits has been present in consumer behavior research since last century [8]. Situational influences were seen as moderating consumer choice. This close correspondence between situational factors and product attributes leads to the question: what constitutes situational factors? Belk $[9,10]$ proposed that environmental factors should include all variables not included in the description of persons or products. Ratneshwar [11,12] suggested that usage context plays a key role in consumer problem-solving by impacting the discriminability of choice alternatives, and that the processes involved might vary with situational familiarity. Contextual goals and constraints might help the consumers to discriminate acceptable alternatives from a much larger available set. Even when the situation is relatively unfamiliar and the decision-maker has to take a more constructive approach to evaluating the alternatives, situational constraints might still facilitate discrimination and quick decisions by focusing the consumer's attention on context-relevant product features. In general, the particular features that get the decision-maker's attention are likely to be those that have relevance for the goal context of the ongoing situation [13]. The role of usage context in consumer choice is one of guiding the search for and evaluation of potential solutions. He et al [14] combined a usage context model and a choice model for demand prediction applications. Recent research on usage context in marketing has led to an exhaustive analysis of usage anticipation and new product diffusion in consumer behavior $[15,16]$.

However, the research of usage situational/contextual information in engineering design has made little progress because of the weakness of interdisciplinary marketing-engineering development. Consumer participated interaction design [17], especially in IT products such as software, mobile phones, and navigation systems, started 10 years ago. For other new products or product line design, such marketing and engineering considerations are also highly interdependent. Michalek et al [18,19] developed a process of Analytical Target Cascading (ATC) to link marketing and engineering. In their work, the complex system is decomposed into hierarchical and interrelated marketing-engineering sub-systems, each of which can be analyzed and optimized separately before being coordinated. The ATC model is based on well-established marketing methodologies, such as conjoint analysis, discrete choice modeling and demand forecasting. In the domain of hi-tech product design, context-aware systems knowing the activity context and taking it into account for system behavior - are emerging. One context-aware system for mobile cartography was mentioned in 
[20], which used formalization to describe situations and contexts for finding typical context patterns. The concepts of usage context are introduced in design engineering in [21-23], [5]. Green et al. published three successive papers on the subject [21-23], with the goal of forming a comprehensive product design methodology that includes contextual factors. Important first steps in the field were taken, including the definition of key terms and concepts. Usage context, as it relates specifically to products, is defined as the unique combination of application and environment in which a product is used. Furthermore, usage context is framed as one part of a larger usage scenario, which also includes market and customer context. This hints at the key role that all three contexts play in guiding the choice of the customer. During the course of the studies, customers were found to have distinct product preferences under different usage contexts. Additionally, evidence supported the contention that contexts could be differentiated according to their functional attributes. This indicates a link between engineering parameters and perceived usefulness, which occurs under the influence of different usage contexts. Yannou et al. [5] presented a Usage Coverage Model (UCM) designed to produce a more thorough marketing model based on sets of permitted usages for a product or a service instead of the conventional perceived marketing attributes. A taxonomy of variables was suggested to set up the link between the design parameters of a product or a service and the part of a set of expected usages that may be covered. The concept of quantified individual performances during usage was proposed, offering the advantage of making links with user experience so that the perceived quality of a product's service could be taken into account. In a further paper [Yannou and Wang, 24], the UCM concept was applied to a common power tool product: a jigsaw. The physics describing the behavior and, consequently, the performances of a jigsaw under given usage contexts were established. When users choose to buy an adapted jigsaw, they may imagine different usage scenarios in which the product may be applied. The usage coverage index proposed [24] revealed whether the product fulfilled customer requirements and expectations.

In the design/redesign process, ingenious and accurate indices are intuitive and help designers make decisions. Several heuristic product family indices have been developed by academics and applied to industry. Most engineering research focuses on the comparison between component and process: Thevenot and Simpson [25] undertook a thorough comparison for heuristic indicators used over a long time period, and then, based on the Commonality vs. Diversity Index [26], they proposed a comprehensive metrics for evaluating commonality [27]. In the evaluation of consumer preference for products, market research methods such as conjoint analysis and discrete choice analysis are utilized [28,29]. Heuristic and statistic-based conjoint data is always indispensable for the near-optimal product family selecting level of consumer attributes [30]. More recently, Luo et al. [31] combined conjoint analysis with GA optimization. They analyzed usage contextual preference distinction using a "robust criteria" analytical method, which limits, in the product's usage environment, the uncontrolled variations of engineering parameters within a robustness criteria $\Delta f$. Since product family optimization turns out to be a combinatorial problem, the meta-heuristic algorithms, such as G.A. [32,33], work relatively well with these pre-studied conjoint analysis data. Belloni et al. [34] recently analyzed and compared several heuristic and A.I. algorithms for a product line design problem. Constraint satisfaction technique works well in solving Constraint Satisfaction Problem [35] and in propagation techniques. This is a convenient and powerful way to model 
uncertainty of design variables and to proceed to domain reductions for discrete variables as well as real variables [36] - one also speaks of interval arithmetics. This is an ideal way to mathematically support the set-based design principles of Toyota (see [37,38]) of a continuously but irremediably uncertainty shrinking process along the design process. In our recent work [39], a consumer usage scenario map was built to represent usage variety in the target market. Usage Coverage Index (UCI) metric was extended to a given product family in the form of a matrix. A practical definition of indicators, economical or efficient, was introduced for the jigsaw cutting wooden board usage.

Based on such previous research, the primary goal of the present research is to extend the measurement of a scale-based product family to individual performance constraints, proposing several indicators for consumer choice. Despite the prosperous research concerning usage context models and consumer preference analysis, little research has given explicit and objective indicators for product family evaluation with regard to target usage contexts and multiobjectives of expected performances. In this paper, we apply a usage context model to model jigsaw product family usages. A set-based comparison between simulated performances and usage satisfaction is employed to measure the feasible usages under consumer usage constraints. Trade-off indicators for different users with diverse usage scenarios and the given product family are compared.

\section{Usage model and usage coverage index}

Our objective is to deliberately put the emphasis on the adequacy of a set of product usage scenarios with given products. This degree of fit or adequacy is referred to as usage coverage [5]. In this paragraph, the concept of usage model and an inference mechanism for determining the usage coverage of a product regarding a set of expected usage scenarios will be presented.

\subsection{Nomenclature}

As the literature review showed, previous works have defined lists of variables of Usage Context Based Design framework [5,24,39]. The following list illustrates the principal notations used in this paper. A jigsaw product family evaluation problem is used as an example throughout this section.

$$
\begin{aligned}
& U \text {-usage context } \\
& k \text {-index for the kth product in the family, } k=1, \ldots, K \\
& i \text {-index for the ith user, } i=1, \ldots, M \\
& j-\text { index for the } j \text { th usage context, } j=1, \ldots, N_{i} \\
& N_{i} \text { - total usage context for user } i \\
& E_{i j} \text { - jth usage context attributes for user } i \\
& w_{i j} \text { - occurrence frequency of usage context } j \text { for user } i \\
& C_{s}-\text { customer attributes affect performance } \\
& X-\text { product design variables } \\
& Y-\text { engineering performance } \\
& \text { Pr - price of the product }
\end{aligned}
$$




\subsection{Usage Context Model and Usage Coverage}

\section{Basic principles}

In our usage model, a product, defined by its design parameter vector $X$, must be adapted as well as possible to a set of customer usages $U$. "Usage needed" is a set of expected usage contexts $E_{i}$ associated with a normalized usage occurrence frequency $w_{i}$, which represents, a percentage of occurrences of given usage context in a year, as defined in formula (1).

$U_{\text {needed }}=\left\{\left(E_{i}, w_{i}\right)\right\}$ with $\sum w_{i}=1$

Given a product design $X$ and a user with certain expertise $C_{s}$, we figure out that the performances of the service are explicitly affected by the user and his/her experience with the product. So performance estimation formulas are required in the form of formula (2).

$Y=f\left(X, E, C_{s}\right)$

When the performances reach certain basic criteria - such as capable for meeting the given service requirement, we say that this usage is feasible with given product by the user. Thus, only a subset of this "usage needed" set may be fulfilled by a given product and user. This part (see figure 2) is called "feasible usage" and is defined in formula (3), where the initial usage needed is expressed as $U_{\text {needed }}=\left\{\left(E_{i}, w_{i}\right)\right\}$. And only a subpart of this usage context $E_{i}^{*} \subseteq E_{i}$ can been done or "covered" for design $X$.

$U_{\text {feasible }}\left(X, U_{\text {needed }}, C_{s}\right)=\left\{\begin{array}{c}\left(E_{i}^{*}, w_{i}\right), \text { such that } \\ \left(E_{i}^{*}, w_{i}\right) \in U_{\text {needed }} \\ \text { and } E_{i}^{*} \subseteq E_{i} \\ \text { and } Y_{i}=f\left(X, E_{i}^{*}, C_{s}\right) \text { is feasible }\end{array}\right\}$

\section{Jigsaw cutting wood usage example}

As an example, a performance estimation model for a jigsaw, based on physical attributes, is used as shown in the appendices. Different categories of variables and a detailed list of intermediate variables for jigsaw design problems are illustrated and interpreted. Hereafter the most important variables for cutting wooden board usage are cited. In practice, the usage context variables in (4) that influence the performance of wood cutting are of the thickness of the wooden board and its density.

$E=\left\{\begin{array}{c}T_{\mathrm{c}}-\text { Thickness of the wood board } \\ \rho_{w}-\text { Density of wood }\end{array}\right.$

We consider two demographic variables $C_{s}=\{$ Gender, Skill $\}$ which are userrelated parameters that affect performances.

$C_{s}=\left\{\begin{array}{c}\text { Gender }- \text { Gender of the saw user } \\ \text { Skill }- \text { Skill of the user for cutting wood with a tool X }\end{array}\right.$

These two variables define the maximal allowable bounds $F_{t \max }$ - the translation force, $F_{p \max }$ - the pressure force, and $M_{w \text { max }}$ (the torque the user's wrist may deliver to the jigsaw). An assessment of these bounds with ergonomic analysis gives the correspondence Table 1 . 
Table 1 Correspondence table between gender and corresponding maximal force and torque admissible values

\begin{tabular}{|c|c|r|r|r|}
\hline Gender & Skill & $F_{t \max }$ & $F_{p \max }$ & $M_{w \max }$ \\
\hline \multirow{4}{*}{ Female } & Basic user & $45 \mathrm{~N}$ & $90 \mathrm{~N}$ & $20 \mathrm{~N} . \mathrm{m}$ \\
\cline { 2 - 5 } & Medium & $80 \mathrm{~N}$ & $130 \mathrm{~N}$ & $40 \mathrm{~N} . \mathrm{m}$ \\
\cline { 2 - 5 } & Professional & $110 \mathrm{~N}$ & $170 \mathrm{~N}$ & $60 \mathrm{~N} . \mathrm{m}$ \\
\hline \multirow{3}{*}{ Male } & Basic user & $70 \mathrm{~N}$ & $105 \mathrm{~N}$ & $30 \mathrm{~N} . \mathrm{m}$ \\
\cline { 2 - 5 } & Medium & $100 \mathrm{~N}$ & $150 \mathrm{~N}$ & $50 \mathrm{~N} . \mathrm{m}$ \\
\cline { 2 - 5 } & Professional & $130 \mathrm{~N}$ & $195 \mathrm{~N}$ & $70 \mathrm{~N} . \mathrm{m}$ \\
\hline
\end{tabular}

We focus on the two essential performance criteria for this usage of "to cut wooden boards" (see formula 6). The first one is the mean advance speed; a nonnull advance speed means that the tool is able to complete this service (feasible). The second one is the comfort during the cutting operation.

$Y=\left\{\begin{array}{r}S_{a}-\text { Mean advance speed of cutting } \\ P_{\text {comfort }}-\text { Degree of comfort in the user's wrist }\end{array}\right.$

The comfort of cutting with a jigsaw is mainly due to the wrist torque which must not exceed a maximal value the user can afford. It can be expressed by:

$P_{\text {comfort }}=1-\left|\frac{M_{w}}{M_{w \text { max }}}\right| \in[0,1]$

\subsection{Definition of Usage Coverage Index (UCl) for Single Usage}

In order to apply computation, the former typological variables describing the usage context must be reinterpreted as intermediate value variables via several correspondence tables. For the usage context aspect, when collecting usage context and user information through a questionnaire [14] or when interpreting intermediate variables, uncertainties are generated because of linguistic ambiguity. A set-based modeling method is used to model the subsets of usage and user contexts. These subsets are modeled by domain variables: discrete sets or continuous intervals.

As illustrated in Fig. 2 below, for a potential usage target market segment, the usage scenario can be identified as $\left(U_{\text {needed }}, C_{S}\right)$; this can be represented as a domain in a Cartesian space formed by the variables defining the usage context ( $E_{1} \times E_{2}$ for illustrative purposes). Given a product design $X$ combined with demographic variables $C_{s}$, its capable usage context can be mapped by a physical-based performance prediction model.

We can also consider the target market as an initial usage context domain. An implemented product design $X$ serves as a set of constraints for the initial usage context domain, because of the limited feasibility and even user exigency on cutting speed and comfort. This initial usage context domain can therefore be considered as a feasible usage context domain: in figure 2 , it is the overlap between initial usage context domain and a given product's capable usage context domain. Alternatively, if we consider the problem in a product design variables space $X$, the initial usage context variables identified in the target market can serve as constraints to reduce the product's design variables domain. 


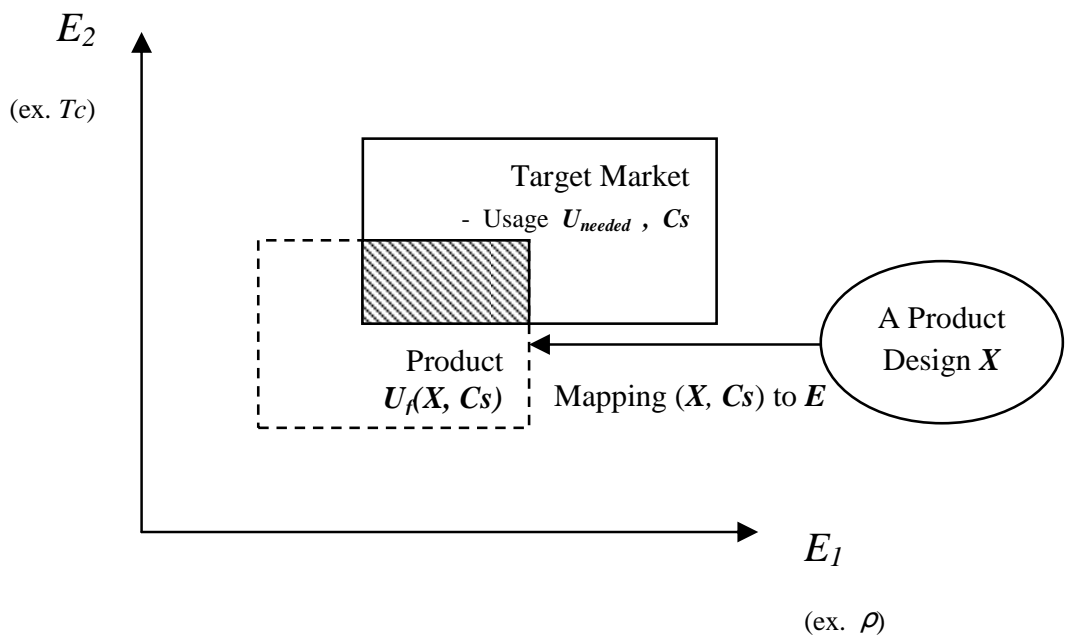

Fig. 2 Usage coverage mechanism in usage context space for single usage

This coverage mechanism cannot be solved properly by mathematical programming, because of the existence of a causal loop in the relation constraints identified in the appendices (Fig. 10). In addition, these variables must be computed in the form of value domains. For these reasons, the usage coverage model forms a Constraint Satisfaction Problem which can be solved by constraint programming techniques.

A constraint satisfaction problem is defined by a triplet $(X, D, C)[35]$ such that:

- $X=\left\{x_{1}, x_{2}, x_{3} \ldots, x_{n}\right\}$ is a finite set of variables that we call constraint variables, $n$ being the number of variables in the problem to be solved.

- $D=\left\{d_{1}, d_{2}, d_{3} \ldots, d_{n}\right\}$ is a finite set of variable value domains of $X$ such that $\forall i \in\{1, \ldots, n\}, x_{i} \in d_{i}$

- $C=\left\{c_{1}, c_{2}, c_{3} \ldots, c_{p}\right\}$ is a finite set of constraints, $p$ being any integer number representing the number of constraints of the problem.

Solving a CSP amounts to finding out the values of each variable of $X$ while at the same time satisfying the set of problem constraints $C$. Over the past years a variety of solving methods have been developed, which enable fast computation of a CSP, and supply the user with intervals containing all the solutions of the CSP. ILOG Solver® provides complete methods for solving CSP problems. The technique used by ILOG Solver is widely known as branch and bound [40].

For our usage context based design problem, the usage context variables $E$ and design variables $X$ are constraint variables, with their initial domains. The constraints are all these physical constraints, feasible constraints, and users' performance exigencies.

After using a constraint programming technique to shrink the intervals of initial usage variables, a possible Usage Coverage Index is defined as the ratio of the final widths of the contracted interval of usage context variables and those of their initial domains, as given in formula (8). The feasibility variable is a Boolean variable, which means that the required usage can be performed to meet basic performance criteria - e.g. a non-null advance cutting speed with no exigency on result quality or comfort in cutting process, for a cutting wooden board usage. 


$$
\begin{gathered}
U C I_{\text {single-usage }}=\text { feasibility } \times \frac{\left|E_{\text {final }}\right|}{\left|E_{\text {initial }}\right|} \times 100 \% \\
=\frac{\prod i \mid \text { usage_context_variable }\left.\right|_{\text {final }}}{\prod i \mid \text { usage_context_variable }\left.\right|_{\text {initial }}} \times 100 \%
\end{gathered}
$$

Here, the reader must pay attention to the fact that we do not deal with the traditional issue of aggregating multiple performances into a sole one. Formula (8) is an exact formula that expresses the degree of domain contraction of variables linked to usage scenarios, in a set-based design approach. Multiplications and ratios are exact ways for resulting in this entire space contraction index.

\subsection{Definition of Usage Coverage Index (UCI) for Multi-Usages}

In daily life, one product may be used in several different situations. Especially for durable products, their usage context can be multiple. We model this situation of multi-usages as a weighted combination of single usage.

We suppose that user $i$ has a set of $j$ usage scenarios for a given product. The different usage scenarios of the user account for $w_{i j}$ in terms of occurrence frequency of usage contexts. As described above, the $U C I$ of each single usage scenario can be calculated. For a given user $i$ with a given product $k$, the $U C I$ of his/her multi-usage context can be calculated using formula (9), which is a weighted sum of all single usages. A more detailed example of multi-usages, the jigsaw case, is illustrated in section 5.3.

$U C I_{\text {multi-usages }}=\sum_{j=1}^{N_{i}}\left(U C I_{i j k} \cdot w_{i j}\right)$ for user $i$ with $N_{i}$ usages

Let us note here that the occurrence frequencies of usage contexts $w_{i j}$ are not consumer preferences. They are thought as a way to compute the coverage of all actual usage situations customers are supposed to face, not the way they aggregate satisfaction regarding reached performances under specific usage situations. The second case supposes that the market is already accustomed to the product type we are presently designing. We have explored this possibility in [14] and in a recent paper [41] where a Discrete Choice Analysis (DCA) method is applied on a customer panel to derive weights in multiple usages and multiple users situations. In our approach, we want to simulate usage coverages even in the presence of innovative products that have still not been launched on the market. It is made possible by a primary computation of a usage scenario space and a further domain contraction of feasible usage scenarios. The discrepancy between both spaces provides a measure of possible usage coverage which is a useful indicator for designers.

Let us also remark that this notion of multiple-usage scenarios has also discussed decades ago in marketing literature. Berkowitz et al. [42] suggested aggregating an individual's given usage situation demand weighted by the situation's frequency of occurrence or importance. While their approach demonstrated the influence of usage suitability on consumer choice, the linkage between usage context and product performance, as well as product design is absent and is still questionable. Indeed, this is not because a usage situation is rare that this use case must not be seriously considered in design. This question is still debated today but we decided to adopt Berkowitz's simplifying assumption. 


\section{Consumer Buying Decision}

The central question for researchers is how consumers respond to various product alternatives. Kotler et al. [43] (Fig. 3) show the stimulus response model of buyer behavior: the marketing and other stimuli enter the consumers' "black box" and generate certain responses. Product, Price and Consumer Characteristics are the factors most concerned by researchers attempting to combine design engineering and marketing. The product design specifications affect consumer preference and consumer characteristics influence how he or she perceives and reacts to the stimuli.

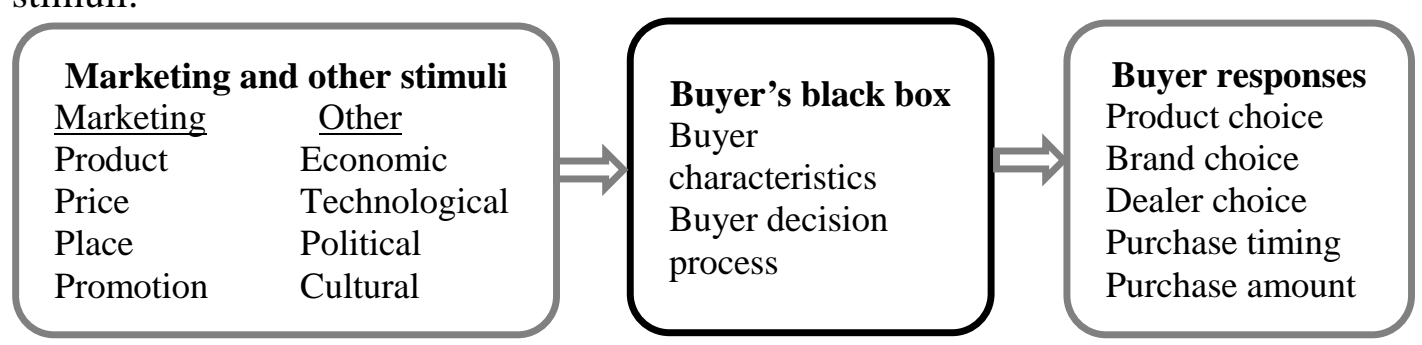

Fig. 3 Model of buyer behavior

For Kotler et al. [43], the buyer decision process consists of five stages: needs recognition, information search, evaluation of alternatives, purchase decision, and post purchase behavior. The alternative evaluation stage is how the consumer processes information in order to come up with a product selection. How consumers evaluate product alternatives usually depends on the individual consumer and the specific buying situation. In certain cases, consumers make careful calculations and think logically, rather than being influenced by subjective aspects like aesthetic, perceptual, or sensorial features, which is also a common situation even for technological products (cars or mobile phones, for instance). In the situation under consideration here, the consumer will mostly be satisfied or dissatisfied in post-purchase behavior (usage). In this stage, consumers utilize the product and the result affects future buying decision and brand loyalty. What determines whether the consumer is satisfied or dissatisfied with a product lies in the relationship between consumer expectations and the product's perceived performances in practical usage situations with a given level of user skills. If the product falls short of expectations, the consumer is disappointed; if it meets expectations, the consumer is satisfied; if it exceeds expectations, the consumer is delighted. However, measurement of the relationship is relatively complicated due to the variety of consumer expectations under different usage contexts. This is a challenging research topic from the designer's point of view. Such measurement during the product design or redesign process is important in design research and has promising potential.

Therefore, based on the marketing proposition of consumer's expectations and product's perceived performances; we define consumer criteria for the decision to buy or not. One criterion is based on the measurement of the adequacy between usage and product. Consumers can make a coverage - efficient compromised choice with the index which increases with perceived performances and adaptation of their expectation, and decreases as product price rises. The values of the performances and price are normalized by the formula (10). This normalization will have a value between $[0,1]: Y_{\text {Max }}$ is the greatest value of $Y$ from all the usage scenarios studied.

$|Y|=\frac{Y}{Y_{\operatorname{Max}}}$ 
The normalized user's decision index $C I$ is given by formula (11) below. It takes the value $[0,1]$ and reflects a compromise decision between expectations, adequacy and economy. The product from all the $P_{i}(i=1, \ldots, K)$ alternatives that produces the maximum $C I$ is the most adapted product for the given usage context and the given user.

$$
\begin{aligned}
& C I=\frac{|U C I| \times \mid \text { Performance } \mid}{\mid \text { Price } \mid} \\
& \text { EfficientCh }(\text { User })=\max _{P_{i}}(C I) \\
& \qquad=\max _{P_{i}}\left(\frac{|U C I| \times\left|S_{\text {a max }}\right| \times\left|P_{\text {com min }}\right|}{\mid \text { Price } \mid}\right)
\end{aligned}
$$

\section{Experimental and simulation results}

\subsection{Power Tool - Jigsaw Family}

In this section, we apply the usage coverage index to check if a given jigsaw product family matches the target usage market well. The expected usage is "to cut wooden boards of different materials and dimensions".

We start with the issue of an existing scale-based family of 4 Bosch jigsaws (from P1 i.e. PST 650 to P4 i.e. Bosch PST900 in table 5), each with increasing power, weight and price. However, their dimensional parameters are the same as

\begin{tabular}{|c|c|c|c|c|}
\hline 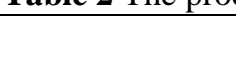 & P1 & $\mathbf{P 2}$ & P3 & P4 \\
\hline Models & PST 650 & PST $700 \mathrm{PE}$ & PST 800 PEL & PST 900 PEL \\
\hline Power $\left(P_{m}\right)$ : & $120 \mathrm{~W}$ & $180 \mathrm{~W}$ & $200 \mathrm{~W}$ & $250 \mathrm{~W}$ \\
\hline Weight $(m)$ & $1.5 \mathrm{~kg}$ & $1.8 \mathrm{~kg}$ & $2 \mathrm{~kg}$ & $2.2 \mathrm{~kg}$ \\
\hline Price $\left(P_{r}\right)$ : & $50 €$ & $80 €$ & $100 €$ & $130 €$ \\
\hline
\end{tabular}
shown in tables 2 and 3.

Table 2 The products in Bosch jigsaw family

Table 3 Jigsaw dimensional design parameters (same values for all products P1 to P4)

\begin{tabular}{|c|l|l|}
\hline \multicolumn{2}{|c|}{ Variables } & \multicolumn{1}{c|}{ Value } \\
\hline$A$ & Blade translation & $0.018 \mathrm{~m}$ \\
\hline$H_{w}$ & Wrist position height & $0.22 \mathrm{~m}$ \\
\hline$L_{w}$ & Wrist position length & $0.09 \mathrm{~m}$ \\
\hline$O_{s}$ & Slider origin position & $0.03 \mathrm{~m}$ \\
\hline$L_{s}$ & Slider length & $0.13 \mathrm{~m}$ \\
\hline$n$ & Number of teeth & 18 \\
\hline$O_{t}$ & Teeth origin position & $0.015 \mathrm{~m}$ \\
\hline$L_{t}$ & Teeth length & $0.068 \mathrm{~m}$ \\
\hline$H_{t}$ & Teeth height & $0.002 \mathrm{~m}$ \\
\hline$W_{t}$ & Teeth width & $0.0012 \mathrm{~m}$ \\
\hline$s$ & Step between two teeth & $0.004 \mathrm{~m}$ \\
\hline$\alpha$ & Rake angle of teeth & $18^{\circ}$ \\
\hline
\end{tabular}

The variables cited in Table 3 Jigsaw dimensional design parameters) can be clearly discerned in figure 10 in the appendix. The relations are established 
between performances $Y$ and $X, E$ and $C_{S}$ through a series of intermediate variables which in this case are mainly forces, torques and speeds. The usage coverage model of the jigsaw design is represented in Fig. below. A physicsbased analysis of these variables is detailed in the appendix parts 2 and 3 .

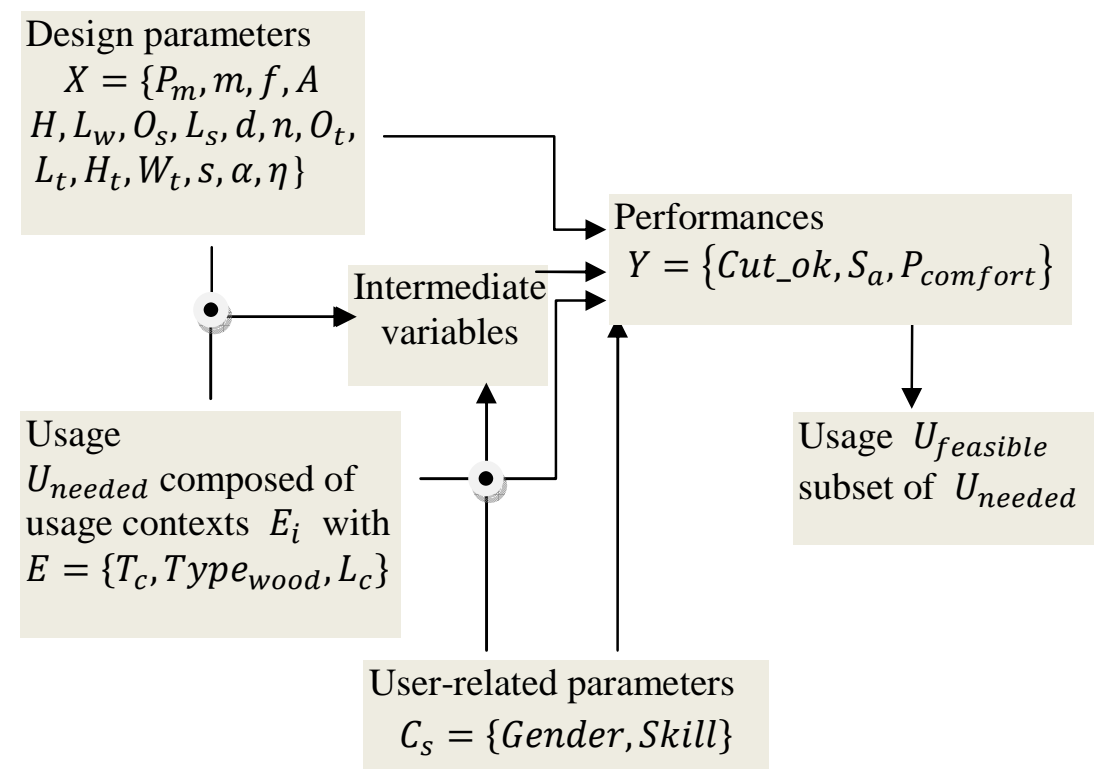

Fig. 4 Variable screening for the jigsaw usage coverage modeling problem

\subsection{Generic usage context space for cutting wooden board usage}

In usage context variable space $\left(T_{c}, \rho\right)$, the difficulty of succeeding the cutting wooden board usage increases as the density and thickness of wooden board increase. The users can probably have a usage of cutting wooden board with density between $[400,900] \mathrm{kg} / \mathrm{m}^{3}$, which is an interval of current natural wood densities. And typical board thicknesses lie within [0.02, 0.05] $\mathrm{m}$. The given jigsaw products P1, P2, P3, P4 have different performances for a given user type.

We suppose that in the target market there are 6 types of typical users: Female Beginner (U0), Female Medium (U1), Female Professional (U2), Male Beginner (U3), Male Medium (U4), and Male Professional (U5). Each user type may not know exactly what kind of usage situation he/she would encounter when cutting wooden board. So, the calculation is based on the entire possible usage context space - in this case, $[400,900] \times[0.02,0.05]$.

\subsubsection{Product family assessment under $\mathrm{UCl}$ criterion}

Under the usage coverage index, value increases as the class of product increases. For the above usage context space, a process of discretization is applied. The process takes the granularity steps of $10 \mathrm{~kg} / \mathrm{m}$ for density and of 0.001 for thickness.

The graphic of coverage is shown in figure 5 after calculation of the $U C I$ index. 


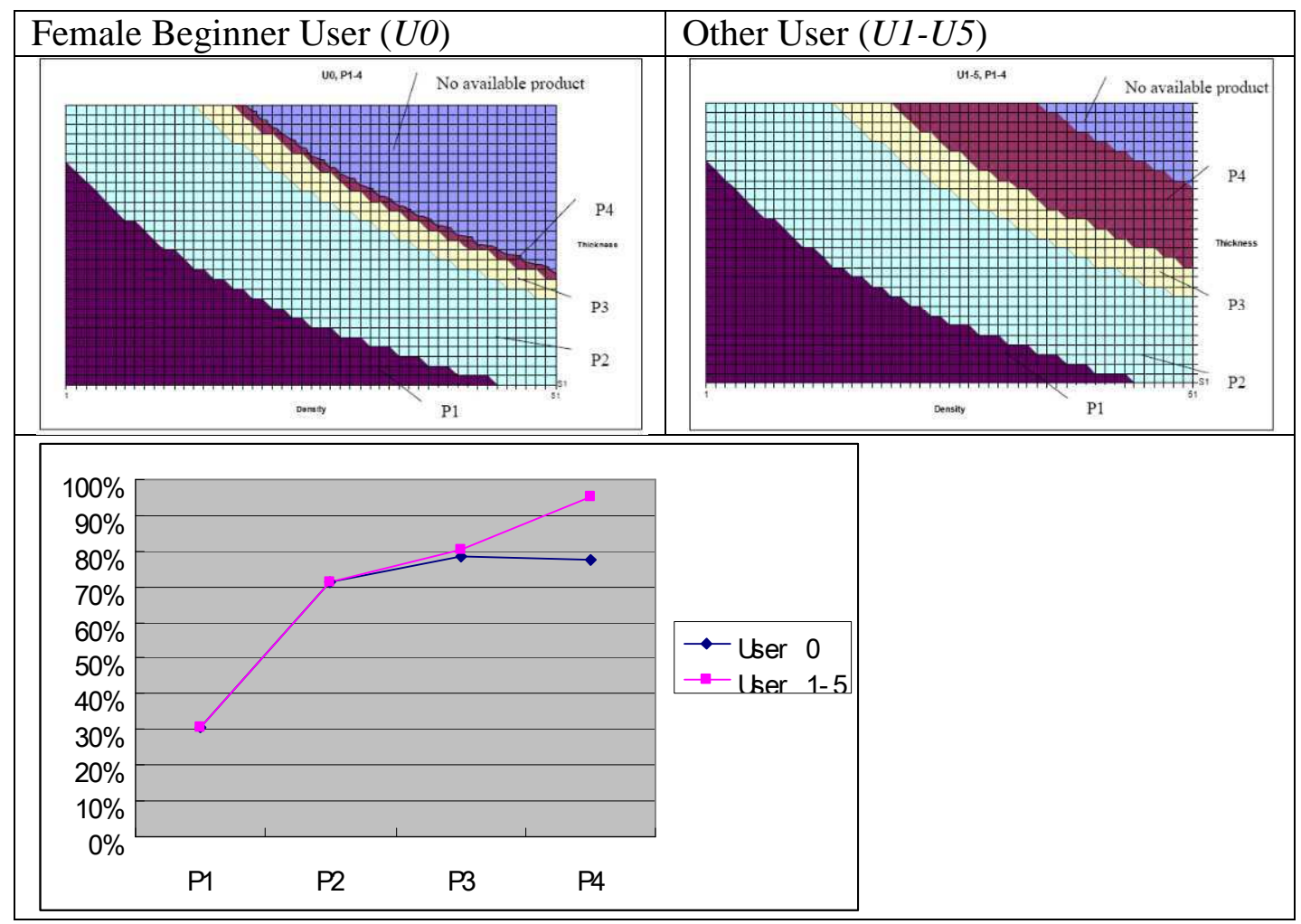

Fig. 5 Illustration of usage context coverage increase with the power capacity of the product family

For example, from the above curve in figure 5 and data of Table 4, it can be seen that product $\mathrm{P} 1$ covers $30.41 \%$ of the usage contexts of given user types. This value increases to $71.17 \%$ for product P2. However, products P3 and P4 see respectively increases of around $10 \%$ and $15 \%$ compared to their predecessors, $\mathrm{P} 2$ and $\mathrm{P} 3$. For a female beginner user in particular, the maximum forces that she can deploy are already at their maximum and thus limit the usage context coverage, which means that she would not be able to succeed in the extremely difficult usage scenarios even with a more powerful product. So the UCI of the most powerful product $\mathrm{P} 4$ does not increase at all compared to $\mathrm{P} 3$. So under $U C I$ criteria, for most user types, product $\mathrm{P} 2$ can satisfy most of their usage contexts. For skilled users, the increasing UCI by product $\mathrm{P} 3$ to $\mathrm{P} 2$ adds little for that by $\mathrm{P} 4$ to $\mathrm{P} 2$.

Table $4 U C I$ values for 6 user types and 4 products

\begin{tabular}{|l|l|l|l|l|l|l|}
\hline UCI & Female & \multicolumn{2}{l|}{ Male } \\
\hline & $\begin{array}{l}\text { User0 } \\
\text { Beginner }\end{array}$ & $\begin{array}{l}\text { User1 } \\
\text { Medium }\end{array}$ & $\begin{array}{l}\text { User2 } \\
\text { Prof. }\end{array}$ & $\begin{array}{l}\text { User3 } \\
\text { Beginner }\end{array}$ & $\begin{array}{l}\text { User4 } \\
\text { Medium }\end{array}$ & $\begin{array}{l}\text { User5 } \\
\text { Prof. }\end{array}$ \\
\hline P1 & $30.41 \%$ & $30.41 \%$ & $30.41 \%$ & $30.41 \%$ & $30.41 \%$ & $30.41 \%$ \\
\hline P2 & $71.17 \%$ & $71.17 \%$ & $71.17 \%$ & $71.17 \%$ & $71.17 \%$ & $71.17 \%$ \\
\hline P3 & $78.26 \%$ & $80.29 \%$ & $80.29 \%$ & $80.29 \%$ & $80.29 \%$ & $80.29 \%$ \\
\hline P4 & $78.44 \%$ & $95.00 \%$ & $95.00 \%$ & $95.00 \%$ & $95.00 \%$ & $95.00 \%$ \\
\hline
\end{tabular}

Although some of the UCI values are identical for a given product with different user types, the other performances, such as the maximum advance speed which can be attained by the user, are not identical. For example, the maximum advance speed $S_{a \text { Max }}$ with product P1 is shown below for the 3 types of female user. Even if the $U C I$ stays the same, $S_{a}(\mathrm{~m} / \mathrm{s})$ performance varies.
User Type 0, P1
User Type 1, P1
User Type 2, P1 

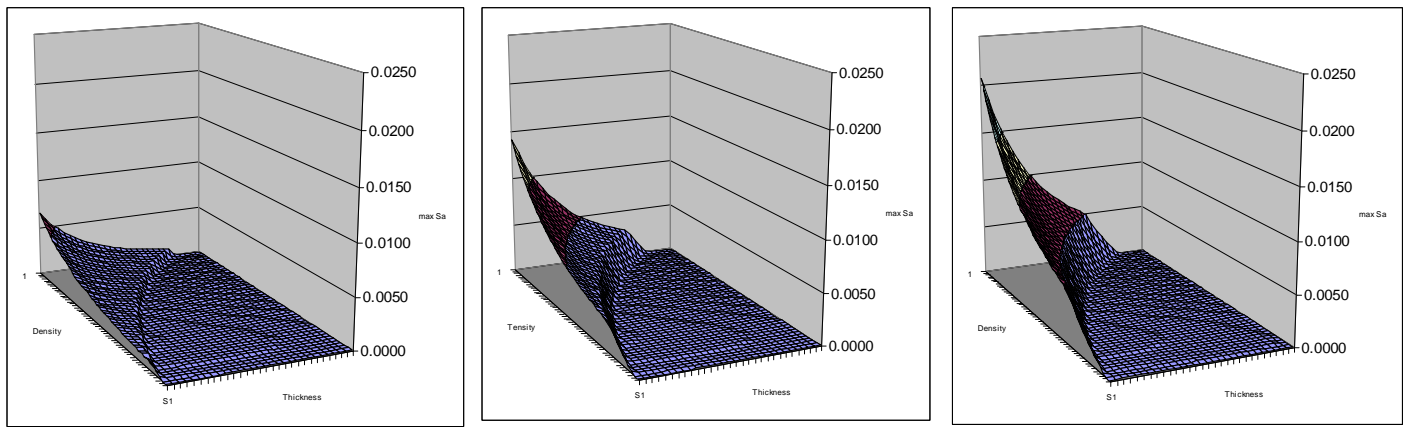

Fig. 6 -Variation of maximum advance speed $S_{a}(\mathrm{~m} / \mathrm{s})$

\subsubsection{Product family assessment under consumer choice}

The decision index $C I$ value of a user type Male Professional with a jigsaw P1 is illustrated in Fig. 7.

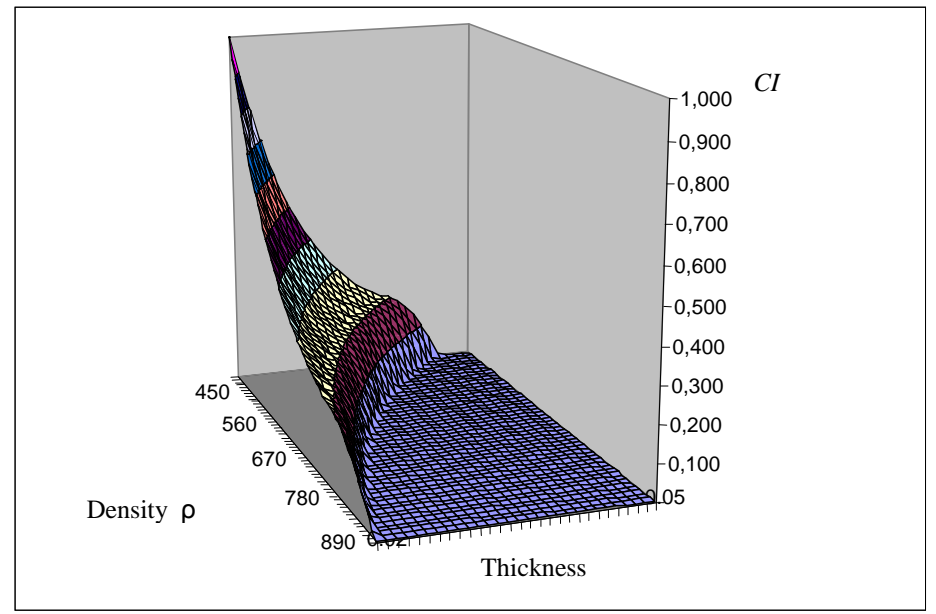

Fig. 7 Decision index for entire usage context space - Male professional user and jigsaw P3

The typical users' decision to choose an appropriate jigsaw of a given product family is based on an average value of the decision index $C I$, as shown in formula (12), from their entire possible usage context space as shown in Fig. 7 above. We refer to this as the Density-Thickness space. In formula (12), all decision index CI's on discrete usage scenarios in usage context space are integrated - under a hypothesis of equal weight for normalized usage context space, since here we consider that the micro usage scenarios are not discriminated by user type.

$C I_{U}=\frac{\int_{\rho} \int_{T_{C}} C I \cdot d T_{C} d \rho}{\int_{\rho} \int_{T_{C}} d T_{C} d \rho}=\frac{\int_{400}^{900} \int_{0.02}^{0.05} C I \cdot d T_{c} d \rho}{500 \times 0.03}$

Table 5 User decision index $(C I)$ for 6 user types in target market

\begin{tabular}{|c|c|c|c|c|c|c|}
\hline \multirow[b]{2}{*}{$C I_{U}$} & \multicolumn{3}{|c|}{ Female } & \multicolumn{3}{|l|}{ Male } \\
\hline & $\begin{array}{l}\text { User0 } \\
\text { Basic }\end{array}$ & $\begin{array}{l}\text { User1 } \\
\text { Medium }\end{array}$ & $\begin{array}{l}\text { User2 } \\
\text { Prof. }\end{array}$ & $\begin{array}{l}\text { User3 } \\
\text { Basic }\end{array}$ & $\begin{array}{l}\text { User4 } \\
\text { Medium }\end{array}$ & $\begin{array}{l}\text { User5 } \\
\text { Prof. }\end{array}$ \\
\hline P1 & 0 & 0.0157 & 0.0653 & 0 & 0.0372 & 0.0856 \\
\hline $\mathrm{P} 2$ & 0 & 0.0123 & 0.0763 & 0 & 0.0340 & 0.1057 \\
\hline P3 & 0 & 0.0109 & 0.0677 & 0 & 0.0301 & 0.0947 \\
\hline $\mathrm{P} 4$ & 0 & 0.0104 & 0.0644 & 0 & 0.0288 & 0.0908 \\
\hline Choice & no & P1 & P2 & no & P1 & P2 \\
\hline
\end{tabular}


Under the integrated decision index values, the most adequate products to target user types are listed in Table 5. No product among the given product family is appropriate for the two beginner user types.

For the two types of beginner user (User0, User3), these is no choice under usage coverage, performance and economic tradeoff criteria, because the maximum torque in a user's wrist is always attained if maximum advance speed is desired.

\subsubsection{Conclusion of the two assessments}

According to the assessment from 5.2.1, the given product family is not well positioned in relation to the 6 types of user in the market. Product P3 may be excluded from the family, because the gain of usage context coverage by P3 to P2 is less significant by that of P4 to P2. P1, P2, P4 serve the target market well. And $\mathrm{P} 4$ is especially useful for skilled users.

Concerning perceived performances and the economic factors, discussed in 5.2.2, the 6 types of user would prefer products P1 and P2 for their generic usage context.

\subsection{Simulated panel of users with usage scenarios}

In this section, the user's usage scenarios are represented as discrete domains, for example a panel of users with different usage scenarios, facing $K$ products in a family which perform the same service with minor distinctions between them. This representation reveals a potential target usage market.

These typical usages in the market are represented as a structure of usage context map. Each user is defined by a set of usage scenarios. Users are supposed to be representative of the market. The usages for each user are weighted with a occurrence frequency of usage context $w_{i j}$. Table 6 gives an example of $M$ users; each one gets $N_{M}$ usage scenarios.

Table 6 Consumers' Usage scenario Map

\begin{tabular}{|c|c|c|c|c|}
\hline User Id & $\operatorname{Usage}_{i, 1}$ & $\boldsymbol{U s a g e}_{i, 2}$ & $\ldots$ & $\boldsymbol{U s a g e}_{i, N i}$ \\
\hline User 1 & $\mathrm{E}_{11}\left(\mathrm{w}_{11}\right)$ & $\mathrm{E}_{12}\left(\mathrm{w}_{12}\right)$ & $\ldots$ & $\mathrm{E}_{1 \mathrm{~N} 1}\left(\mathrm{w}_{1 \mathrm{~N} 1}\right)$ \\
\hline User 2 & $\mathrm{E}_{21}\left(\mathrm{w}_{21}\right)$ & $\mathrm{E}_{22}\left(\mathrm{w}_{22}\right)$ & $\ldots$ & $\mathrm{E}_{2 \mathrm{~N} 2}\left(\mathrm{w}_{2 \mathrm{~N} 2}\right)$ \\
\hline User 3 & $E_{31}\left(w_{31}\right)$ & $\mathrm{E}_{32}\left(\mathrm{w}_{32}\right)$ & $\ldots$ & $\mathrm{E}_{3 \mathrm{~N} 3}\left(\mathrm{w}_{3 \mathrm{~N} 3}\right)$ \\
\hline $\begin{array}{c}\cdots \\
\text { User } M\end{array}$ & $\mathrm{E}_{\mathrm{M} 1}\left(\mathrm{w}_{\mathrm{M} 1}\right)$ & $\mathrm{E}_{\mathrm{M} 2}\left(\mathrm{w}_{\mathrm{M} 2}\right)$ & $\ldots$ & $\mathrm{E}_{\mathrm{MNM}}\left(\mathrm{w}_{\mathrm{MNM}}\right)$ \\
\hline
\end{tabular}

The numbers of different usages $N_{i}$ for a use $i=1, \ldots, M$ may vary for the different users $i$. And the relative weights of each usage context should respect equation (13).

$\sum_{j=1}^{N_{i}} w_{i j}=1$, with $i=1, \ldots, M$

For each Product $P_{k}$ and user $i$, a series of $N_{i}$ user decision indices $(C I)$ and usage context coverage indices $(U C I)$ is then calculated. And a total $C I, U C I$ for user $i$ 's multi-usages $N_{i}$ by a product $P_{k}$ can be calculated using formula (14).

$C I_{i k}=\sum_{j=1}^{N_{i}}\left(C I_{i j k} w_{i j}\right)$, with $i=1, \ldots, M$ 
For example: If a Female Basic User wants to cut a hard wooden board (such as oak) of $0.035 \mathrm{~m}$ thickness, a medium wooden board (such as pine) of $0.050 \mathrm{~m}$ thickness, and a soft wooden board (such as plywood) of $0.015 \mathrm{~m}$ thickness, each usage scenario will be given relative weighted importance. She has 4 Bosch jigsaws listed in table 4 to choose from.

If we consider these 3 usage scenarios with usage occurrence frequencies $w_{1}, w_{2}, w_{3}$, then this user will choose the product with a maximum composite decision index $C I$, defined by formula (15).

$\operatorname{Choice}\left(P_{k}\right)=\max _{P_{i}}\left(\sum_{j=1}^{3}\left(C I_{j k} w_{j}\right)\right)$, with $k=1,2,3,4$

\subsubsection{Results and conclusion of simulations}

For experimental illustration, we randomly generate a panel of 100 users from 6 different types, using a combination of gender and skill in $C_{s}$ variables as listed in section 3.2. Each of the users has at most 6 usages with different weights. The usages are also generated with 3 types of wood (soft, medium, hard) and with a thickness which is uniformly distributed in the interval $[0.010,0.060] \mathrm{m}$. A userusages map is generated randomly.

The user's decision to choose an appropriate jigsaw for his/her potential composite usages is based on the index shown in section 5.2.2.

The given Bosch Jigsaw product family, whose features were listed in Table 2, is used as a reference in Table $7-100 \%$ of power, weight, and price. We can see that, for a generally uniform-distributed usage scenarios case, the given jigsaw product family corresponds to the target usage market well: P1 takes $30 \%$ of the market share, P2 41\%, P3 6\%, and P4 17\%, with only 6\% of users unable to find an appropriate jigsaw for their specific usage scenarios. Products P1, P2, and P4 take in total $88 \%$ of the market share, while P3 is redundant, which was the prediction in section 5.2.3.

Table 7 Products' usage market share estimation

\begin{tabular}{|c|c|c|c|c|}
\hline & Power, Weight, Price & $50 \%$ & $100 \%$ & $150 \%$ \\
\hline \multirow[t]{3}{*}{ P1 } & Average Decision Index $(\mathrm{Cl})$ & 0.022 & 0.141 & 0.164 \\
\hline & Average Usage Coverage Index (UCl) & 0.035 & 0.300 & 0.522 \\
\hline & User Choice & 3 & 30 & 61 \\
\hline \multirow[t]{3}{*}{ P2 } & Average Decision Index $(C)$ & 0.099 & 0.156 & 0.158 \\
\hline & Average Usage Coverage Index (UCl) & 0.166 & 0.522 & 0.698 \\
\hline & User Choice & 24 & 41 & 32 \\
\hline \multirow[t]{3}{*}{ P3 } & Average Decision Index $(\mathrm{Cl})$ & 0.101 & 0.141 & 0.138 \\
\hline & Average Usage Coverage Index (UCl) & 0.211 & 0.574 & 0.732 \\
\hline & User Choice & 3 & 6 & 0 \\
\hline \multirow[t]{3}{*}{ P4 } & Average Decision Index $(C l)$ & 0.117 & 0.137 & 0.123 \\
\hline & Average Usage Coverage Index (UCl) & 0.321 & 0.671 & 0.755 \\
\hline & User Choice & 54 & 17 & 4 \\
\hline $\mathrm{x}$ & Users do not choose & 16 & 6 & 3 \\
\hline
\end{tabular}

For illustrative purposes, we generate two fictive product families, scaled down or up respectively by $50 \%$ and $150 \%$ of the power, weight and price of the given Bosch Jigsaw product family. They can be considered as competing or alternative jigsaw product family compositions. The former consists of less powerful and less expensive products. The latter is, conversely, more powerful and more expensive. 
For the given target usage market - represented by the user panel - the question is whether the Bosch Jigsaw family composition is well composed or not.

For a less powerful product family (scaled down by 50\%), the percentage of users whose usage scenarios have no adaptive choice in the family increases from $6 \%$ to $16 \%$. The given panel of users shifts for more powerful products $\mathrm{P} 2, \mathrm{P} 4$ as shown in Fig. 8. For the case of more powerful product family (scaled up to $150 \%$ ), firstly, the increase of no choice users is less significant; secondly, the more powerful products $\mathrm{P} 3$ and $\mathrm{P} 4$ are less preferred due to their higher price.

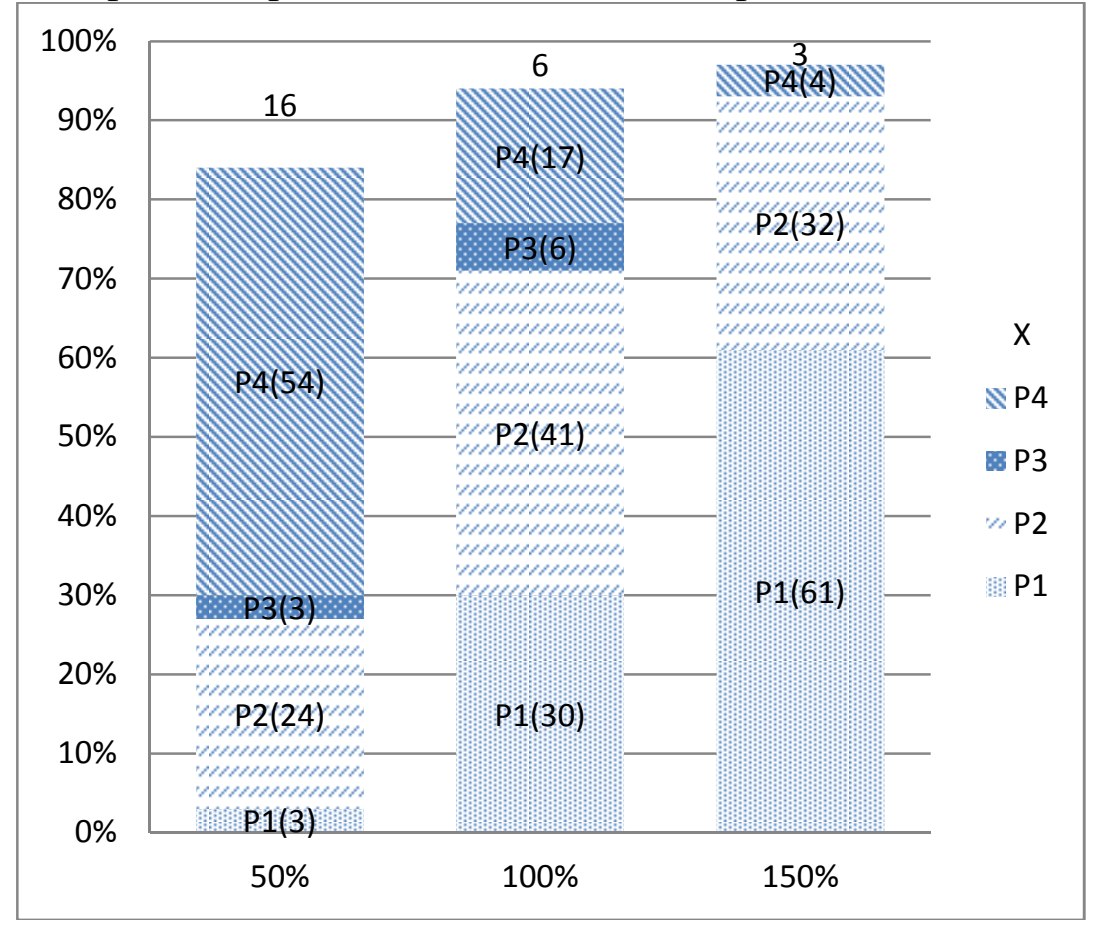

Fig. 8 The usage market shares for the jigsaw families

The above comparison reveals that the Bosch Jigsaw family studied covers the target usage market quite well; however, since the product P3 is too close to P2 and P4's performances (similar specification) and P2 has better usage coverage and performances for its price, and since $\mathrm{P} 4$ is more powerful for extremely hard usages, product $\mathrm{P} 2$ and $\mathrm{P} 4$ cannibalize the market part of $\mathrm{P} 3$. A better composition of products in the family can be further studied in regard to the target usage market.

Similarly, we take the extreme cases - User type 5 (male professional user) and User type 0 (female basic user); each user type will face easy wooden board cutting usage scenarios (wood type 0 or 1 , thickness drawn uniformly from [0.01, $0.03]$ ) and hard wooden board cutting usage scenarios (wood type 1 or 2 , thickness drawn uniformly from $[0.03,0.06])$. The choice of products of a randomly generated group of 100 typical users with composite usage scenarios is shown in figure 9. 


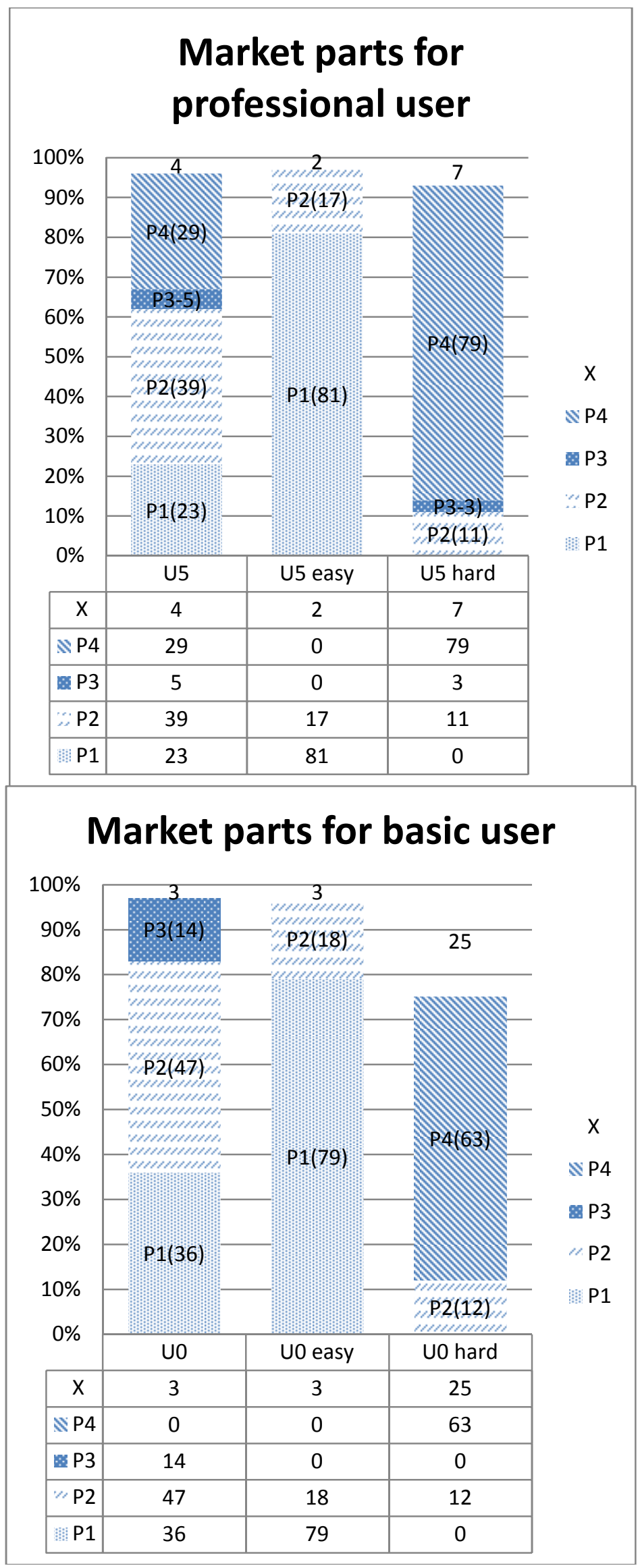

Fig. 9 The market parts for the products in Bosch product family for professional/beginner user In the left-hand chart, we can see, for professional users cutting wooden board usage, they prefer P4 for their difficult usage scenarios, and P1 or P2 for their 
easy usage scenarios. This justifies the existence of product P4. A professional user with all the range of usage scenarios (column 1) may choose any of the three products $\mathrm{P} 1, \mathrm{P} 2$, or $\mathrm{P} 4$.

For the basic users in the right-hand chart, products P1and P2 are the most preferred. $25 \%$ of difficult usage scenarios cannot be served by any product in the family. P3 can also be substituted by either P2 or P4.

\section{Conclusions}

Dickson in 1982 [44] pleaded for a renewal of marketing research in better segmenting by usage situations: "A recent comprehensive state of the art review of market segmentation concluded that the field has become too fixed in its ways and that new conceptualizations of the segmentation problem should be explored. One convention that bears examination is the equating of market segmentation with customer segmentation. Markets can also be subdivided by usage situation. Although almost every conceivable person-based characteristic has been used to segment markets over the last decades, there has been a disturbing lack of consideration of the usage situation as a basis for defining product markets and modeling consumer choice behavior."

Although the concept of usage context information is not new in marketing research, its use in design engineering, especially in helping product family design is relatively void. The presented usage coverage model successfully combines the two aspects of product design process: engineering and marketing researches. The advantage compared to traditional demand estimation in marketing research is to reduce the complexity of survey and data analysis since the product or product family may be totally innovative and does not require to be known beforehand by customers. A primary computation of a usage scenario space and a further simulation of product (and product family performances) under specific usage scenarios with set-based techniques allow us to compute Usage Coverage Indices (UCIs) which express domain contraction - to be feasible - of usage scenario variables. This new physics-based simulation process is summarized in Figure 10.

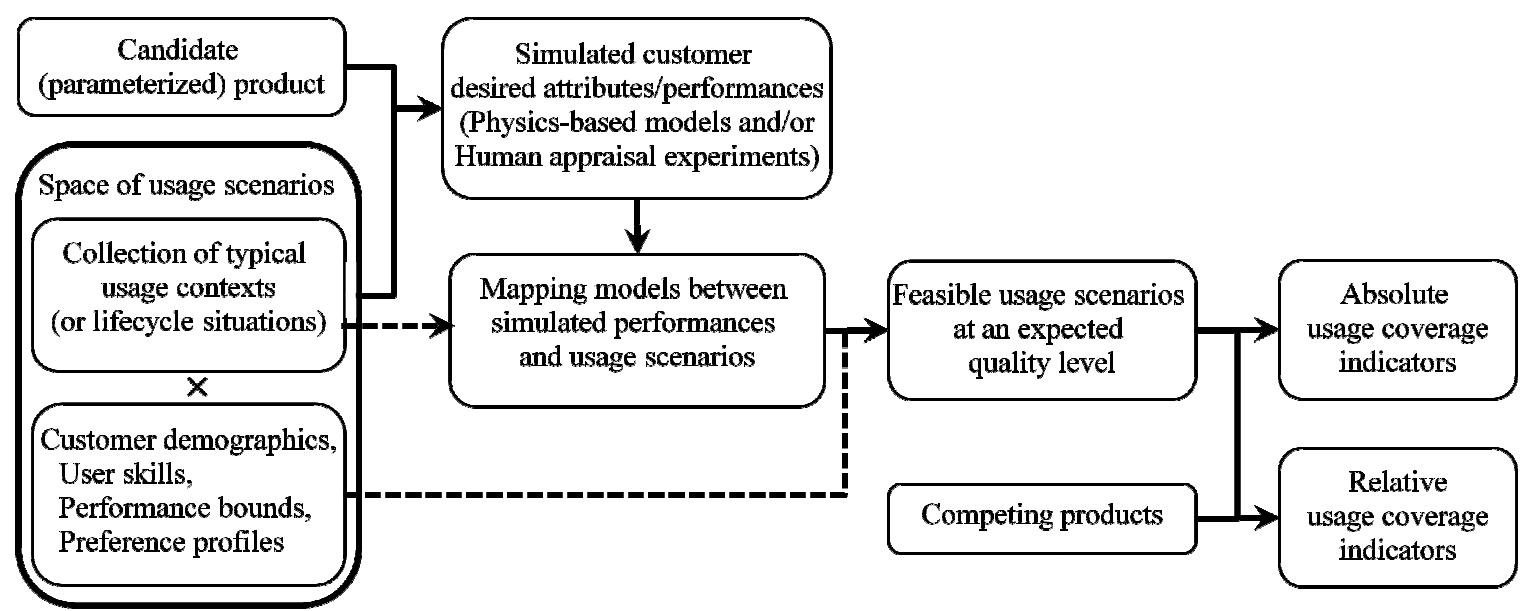

Fig. 10 Our new framework of Set-based Design by usage coverage simulation

The concept of Usage Coverage Index is applied to composite usage scenarios. $U C I$ metric is then extended to a given product family in the form of a matrix. To measure the quality/price tradeoff of users, we propose a user's decision index $C I$. A constraint programming technique is applied in the process of $U C I$ calculation 
and performance estimation. Simulations with a jigsaw family for cutting wood usages are implemented in the case of a product family redesign.

The experimental results show that the proposed indices help to evaluate the adaptability, for a given scale-based product family, to diverse usage scenarios in a target market. Interesting redesign suggestions can be drawn from the given indices and charts: designers can rely on the results to eliminate redundant units in the family. Scale-based configurations of the products can be rapidly simulated and compared to find out an appropriate series. This study clearly demonstrates the usefulness of a usage coverage model based approach to assess the composition and configuration of a product family design.

The limits of the present design process is that a well established physics-based product model is required for the purpose of individual performance calculation. Nonetheless most products can be studied in sufficient detail, using a physicsbased model or a heuristics-based (or human appraisal) model. Relations between usage contexts and performances delivered by a product in these contexts can then often be successfully established.

Forthcoming developments are presently studied. Our model may be used to sample a given product family, considering some other competing families on the market, to calculate more realistic market shares. Lastly, a user interactive product selection platform might be helpful for customers when looking for the best adapted product or service in a store, covering at best his or her usage expectations.

\section{References}

1. Alizon F, Shooter SB, Simpson TW (2007) Henry ford and the model T: lessons for product platforming and mass customization. Paper presented at the ASME International Design Engineering Technical Conferences, Las Vegas, Nevada,

2. Pine BJ (1993) Mass customization, the new frontier in business competition. Harvard Business School Press.

3. Tseng MM, Jiao J (2001) Mass customization, in: Handbook of industrial engineering, technology and operation management. 3 edn. New York, NY: Wiley,

4. Johnson C (2006) Who Buys Custom Consumer Products? Why Custom Product Buyers Could Be Your Most Important Consumers.

http://www.forrester.com/rb/Research/who_buys_custom_consumer_products/q/id/39664/t/2.

5. Yannou B, Chen W, Wang J, Hoyle C, Drayer M, Rianantsoa N, Alizon F, Mathieu J-P (2009)

Usage coverage model for choice modeling: principles. Paper presented the ASME International

Design Engineering Technical Conferences, San Diego, USA,

6. Meyer MH, Lehnerd AP (1997) The power of product platforms: building value and cost leadership. The Free Press,

7. Green EP, Srinivasan V (1978) Conjoint analysis in consumer research: issues and outlook. Journal of Consumer Research 5:103-123

8. Srivastava RK (1981) Usage-situational influences on perceptions of product-markets: theoretical and empirical issues. Advances in Consumer Research 8:106-111

9. Belk RW (1974) An exploratory assessment of situational effects in buyer behavior. Journal of Marketing Research 11 (May):156-163

10. Belk RW (1975) Situational variables and consumer behavior. Journal of Consumer Research 2 (December):157-264

11. Ratneshwar S, Shocker AD (1991) Substitution in Use and the Role of Usage Context in Product Category Structures. Journal of Marketing Research 28 (3):281-295

12. Ratneshwar S, Warlop L (1993) The role of usage context in consumer choice: a problemsolving perspective. Advances in consumer research 20 (377-382)

13. Huffman C, Houston MJ (1993) Goal-oriented experiences and the development of knowledge. Journal of Consumer Research 20 (2):190-207 
14. He L, Hoyle C, Chen W, Yannou B, Wang J (2010) A framework for choice modelling in usage context-based design. Paper presented at the ASME International Design Engineering Technical Conferences, Montreal, Quebec, Canada, August 15-18

15. Shih C-F, Venkatesh (2004) Beyond adoption: development and application of a use-diffusion model. Journal of Marketing 68 (January):59-72

16. Hoffmann J, Roehrich G, Mathieu J-P (2006) The role of anticipation of usage and intention of usage in the evaluation of new product. Paper presented at the AFM conference, Nantes, France, 11, 12 May

17. Bergman E (2000) Information appliances and beyond: interaction design for consumer products. Academic Press,

18. Michalek JJ, Feinberg FM, Papalambros PY (2005) Linking marketing and engineering product design decision via analytical target cascading. Journal of Product Innovation Management 22:42-62

19. Michalek JJ, Feinberg FM, Adiguzel F, Ebbes P, Papalambros PY (2008) Realizable product line design optimization: coordinating marketing and engineering models via analytical target cascading. Marketing Science

20. Reichenbacher T (2003) Adaptive methods for mobile cartograph. Paper presented at the 21st International Cartographic Conference, Durban, South Africa, 10-16 August

21. Green MG, Linsey JS, Seepersad CC, Wood KL (2006) Frontier design: a product usage context method. Paper presented at the ASME International Design Engineering Technical Conferences, Philadelphia, PA, USA,

22. Green MG, Palani RPK, Wood KL (2004) Product usage context: improving customer needs gathering and design target setting. Paper presented at the ASME International Design Engineering Technical Conferences, Salt Lake City, UT., USA, Sept. 28-Oct. 2

23. Green MG, Tan J, Linsey JS, Seepersad CC, Wood KL (2005) Effects of product usages context on consumer product preferences. Paper presented at the ASME International Design Engineering Technical Conferences, Long Beach, CA., USA,

24. Yannou B, Wang J, Yvars P (2010) Simulation of the usage coverage of a given product. Paper presented at the International Design Conference - Design 2010, Dubrovnik, Croatia, May 17 - 20

25. Thevenot HJ, Simpson TW (2006) Commonality indices for product family design: a detailed comparison. J Eng Design 17 (2):99-119. doi:Doi 10.1080/09544820500275693

26. Alizon F, Shooter SB, Simpson TW (2006) Assessing and improving commonality and diversity within a product family. Paper presented at the ASME International Design Engineering Technical Conferences, Philadelphia, Pennsylvania, USA, September 10-13

27. Thevenot HJ, Simpson TW (2007) A comprehensive metric for evaluating component commonality in a product family. J Eng Design 18 (6):577-598. doi:Doi 10.1080/09544820601020014

28. Wassenaar HJ, Chen W (2003) An approach to decision-based design with discrete choice analysis for demand modeling. J Mech Design 125 (3):490-497. doi:Doi 10.1115/1.1587156

29. Wassenaar HJ, Chen W, Cheng J, Sudjianto A (2005) Enhancing discrete choice demand modeling for decision-based design. J Mech Design 127 (4):514-523. doi:Doi 10.1115/1.1897408

30. Kohli R, Sukumar R (1990) Heuristics for product line design using conjoint analysis. Management Science 36 (12):1464-1478

31. Luo L (Forthcoming) Product line design for consumer durables: an integrated marketing and engineering approach. Journal of Marketing Research

32. Balakrishnan PV, Gupta R, Jacob VS (2004) Development of hybrid genetic algorithms for product line designs. Ieee T Syst Man Cy B 34 (1):468-483. doi:Doi 10.1109/Tsmcb.2003.817051

33. Balakrishnan PV, Gupta R, Jacob VS (2005) An investigation of mating and population maintenance strategies in hybrid genetic heuristics for product line designs. Comput Oper Res 33 (3):639-659. doi:DOI 10.1016/j.cor.2004.07.01

34. Belloni A, Freund R, Selove M, Simester D (2008) Optimizing product line design - efficient methods and comparisons. Management Science 54 (9):1544-1552

35. Tsang E (1993) Foundations of constraint satisfaction. Academic Press London and San Diego, 36. Benhamou F, Granvilliers L (2006) Continuous and interval constraints, Handbook of constraint programming. Elsevier,

37. Finch WW (1999) Set-based models of product platform design and manufacturing processes. Paper presented at the ASME International Design Engineering Technical Conferences, Las Vegas, USA,

38. Ward AC, Liker JK, Sobek DK, Cristiano JJ (1994) Set-based concurrent engineering and Toyota. Paper presented at the ASME International Design Engineering Technical Conferences, Sacramento, California, 
39. Wang J, Yannou B (2011) Explicit product family indicators based on a constraint programming simulation of usage coverage. Paper presented at the International Conference on Research into Design, Bangalore, Indian, 10-12 January

40. ILOG (2001) ILOG Solver 5.4 User's Manual. ILOG,

41. He L., Chen W., Hoyle C., Yannou B. (2012) Choice modeling for usage context-based design, Journal of Mechanical Design, in press, DOI: 10.1115/1.4005860.

42. Berkowitz E.N., Ginter J.L. et Talarzyk W.W. (1977) An investigation of the effects of specific usage situations on the prediction of consumer choice behavior, in Educators' Proceedings, B.A. Greenberg and D.N. Bellenger, (Ed.), Chicago : American Marketing Association, in Contemporary Marketing Thought, pp. 90-94.

43. Kotler P, Armstrong G (2009) Principles of marketing. Pearson Education International.

44. Dickson, P. (1982) Person-Situation: Segmentation's Missing Link, Journal of Marketing, 46(4), pp. 56-64. 


\section{Appendices}

\section{Jigsaw parameterization}

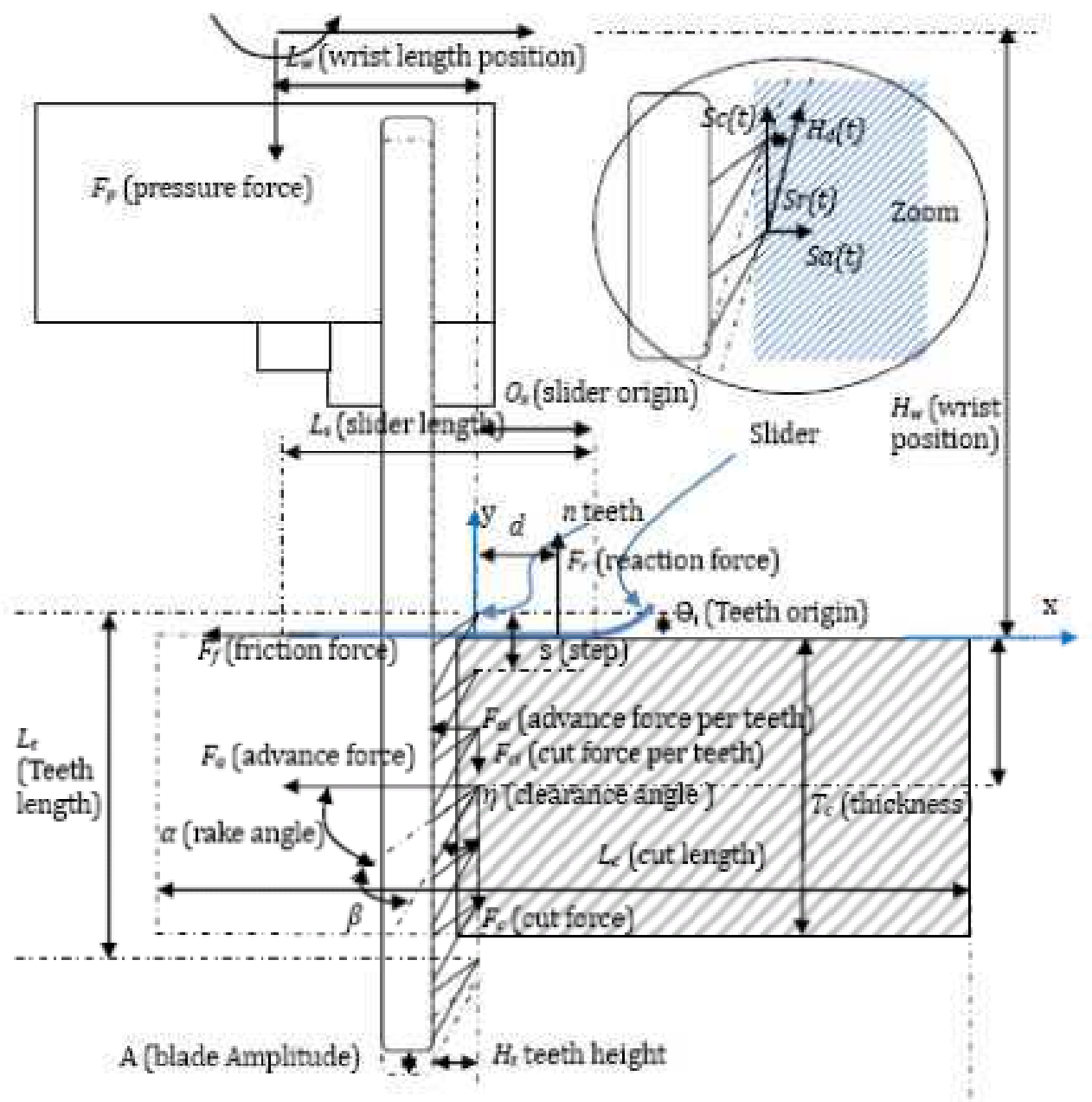

Fig. 11 Jigsaw parameterization: dimensions, forces, torques, speeds 


\section{Intermediate variables for the jigsaw modeling}

\begin{tabular}{|l|l|}
\hline \multicolumn{2}{|c|}{ INTERMEDIATE VARIABLES (Forces, Speed) } \\
\hline$\rho_{w}(\mathrm{~kg} / \mathrm{m} 3)$ & Wood density \\
\hline$\mu($ no unit) & Friction factor between the steel and wood \\
\hline$F_{t}(\mathrm{~N})$ & Translation force \\
$F_{t \text { max }}(\mathrm{N})$ & $\begin{array}{l}\text { Maximal translation force that can be } \\
\text { delivered by the given user defined by } C_{s} \\
\text { vector }\end{array}$ \\
\hline$F_{p}(\mathrm{~N})$ & Pressure force \\
\hline$F_{p \max }(\mathrm{N})$ & $\begin{array}{l}\text { Maximal pressure force that can be delivered } \\
\text { by the given user defined by } C_{s} \text { vector }\end{array}$ \\
\hline$M_{w}(\mathrm{~N} \cdot \mathrm{m})$ & Wrist torque \\
\hline$M_{w}$ max $(\mathrm{N} \cdot \mathrm{m})$ & $\begin{array}{l}\text { Maximal } \text { wrist torque that can be delivered by } \\
\text { the given user defined by } C_{s} \text { vector }\end{array}$ \\
\hline$F_{r}(\mathrm{~N})$ & Reaction force between slider and wood \\
\hline$F_{f}(\mathrm{~N})$ & Friction force between slider and wood \\
\hline$F_{a}(\mathrm{~N})$ & Advance force \\
\hline$F_{a i}(\mathrm{~N})$ & Elementary advance force on tooth $i$ \\
\hline$F_{c}(\mathrm{~N})$ & Cut force \\
\hline$F_{c i}(\mathrm{~N})$ & Elementary cut force on tooth $i$ \\
\hline$H_{d}(\mathrm{~m})$ & Height of scobs \\
\hline$N_{m}$ & $\begin{array}{l}\text { Mean number of teeth cutting wood at any } \\
\text { moment }\end{array}$ \\
\hline$S_{c}(\mathrm{~m} / \mathrm{s})$ & Mean cutting speed \\
\hline$S_{c}(t)(\mathrm{m} / \mathrm{s})$ & Instantaneous cutting speed \\
\hline$S_{a}(t)(\mathrm{m} / \mathrm{s})$ & Instantaneous advance speed \\
\hline
\end{tabular}

\section{Other relations for the jigsaw physics}

\section{Geometrical relations}

In the upper position of the blade, the upper tooth is always above the slider is expressed by:

$$
O_{t}>0
$$

The lower tooth in the upper position is below the lower wood stick plane (teeth are cutting all along the thickness at any moment of the cutting period):

$$
\mathrm{t} \in\left[\frac{1+2 n}{2 f}, \frac{n+1}{f}\right], n \in N \Rightarrow L_{t}-O_{t} \geq T_{c}
$$

The useful length of the blade $\mathrm{L}_{t}$, is proportional to step $\mathrm{s}$ and the number of teeth $\mathrm{n}$ minus 1 :

$$
L_{t}=(\mathrm{n}-1) \cdot \mathrm{s}
$$

\section{Static relations}

All the forces are non-negative (by construction on Figure 11). And the slider is always touching the wood surface. Then: 


$$
F_{r} \geq 0, F_{a} \geq 0, F_{f} \geq 0
$$

The forces $F_{t}$ and $F_{p}$ delivered by the user onto the jigsaw tool can be modeled as intervals of possible values which are bounded by the maximum allowable translation force $\mathrm{F}_{\text {tmax }}$ and pressure force $\mathrm{F}_{\mathrm{p} \text { max }}$ a user can afford:

$$
F_{t} \in\left[0, F_{t_{\text {max }}}\right], F_{p} \in\left[0, F_{p \text { max }}\right]
$$

As previously seen with Table 1, these maximal allowable bounds are dependent from the user gender and the user skill.

The horizontal force equilibrium (advance acceleration is neglected) is expressed by:

$$
F_{a}=F_{t}-F_{f}
$$

In the following, we assume that $F_{t}$ may be considered as a constant entry force, imposed by the user.

The friction force between the jigsaw slider and the wood is expressed by:

$$
F_{f}=F_{r} \cdot \mu
$$

The vertical force equilibrium is expressed by:

$$
F_{p}+m g+F_{c}=F_{r}
$$

The momentum equilibrium at wrist position provides the expression of the wrist torque:

$$
M_{w}=F_{a}\left(H_{w}+\frac{T_{c}}{2}\right)+F_{c} L_{w}+F_{f} H_{w}-\left(d+L_{w}\right) F_{r}
$$

In the same manner, the torque in the user wrist $\mathrm{M}_{\mathrm{w}}$ is bounded by a maximal allowable torque the user may support expressed in table 1.

$$
M_{w} \leq M_{w \max }
$$

The position of the reaction force of the slider must stay inside the slider surface area so as to avoid for the jigsaw tool to flip:

$$
\mathrm{d} \in\left[O_{s}-L_{S}, O_{s}\right]
$$

\section{Cutting technological relations}

The minimal teeth number for a successful cut is 3 :

$$
\mathrm{Tc}>3 s
$$

The mean number of teeth cutting wood at any moment is expressed by:

$$
N_{m}=\frac{T_{c}}{S}
$$

The elementary advance force $\mathrm{F}_{\mathrm{a}_{\mathrm{i}}}$ on a tooth $\mathrm{i}$ is provided by: 


$$
F_{a i}=\frac{F_{a}}{N_{m}}
$$

This equation means that $\mathrm{F}_{\mathrm{ai}}$ is constant. During the cutting phase, when the blade is ascending, a cutting relation exists between the elementary advance force $\mathrm{F}_{\mathrm{a}_{\mathrm{i}}}(\mathrm{t})$ on a tooth $\mathrm{i}$ and the scobs height $\mathrm{H}_{\mathrm{d}}(\mathrm{t})$. As $F_{a i}$ is constant, then $H_{d}$ is constant when the blade ascends and the relation is:

$$
\begin{aligned}
\mathrm{t} \in\left[\frac{1+2 n}{2 f}, \frac{n+1}{f}\right], n \in N \Rightarrow F_{a i} \\
=W_{t} \rho_{w}\left(H_{d} A_{2}+A_{2}\right) e^{A_{1}(35-\alpha)}
\end{aligned}
$$

This equation was found based on experimental measures of $H_{d}$ for different types of wood and teeth (i.e., different values of $\left.\mathrm{W}_{\mathrm{t}}, \rho_{\mathrm{w}}, \mathrm{H}_{\mathrm{d}}, \alpha\right)$. The three $A_{i}$ coefficients have been found experimentally:

$$
A_{1}=0.01, A_{2}=272000, A_{3}=0
$$

Finally, we obtain from (15) and (16) the following relation between $H_{d}$ and $F_{a i}$ :

$$
H_{d}=\frac{F_{a i}}{272000 W_{t} \rho_{w} e^{0.01(35-\alpha)}}
$$

As well, a constraint should be:

$$
H_{d} \leq \mathrm{s}
$$

During the descending phase, the scobs height is zero:

$$
\mathrm{t} \in\left[\frac{n}{f}, \frac{1+2 n}{2 f}\right], n \in N \Rightarrow H_{d}(t)=0
$$

The Elementary cutting force on a tooth during the cutting - ascending - phase is:

$$
F_{c i}=W_{t} \rho_{w}\left(60000 H_{d}+20\right) e^{0.01(35-\alpha)}
$$

This equation has also been found experimentally. In the same manner, the cutting force is not dependent of time.

During the descending phase, the cutting force is primarily due to the friction between the teeth and wood:

$$
F_{c i}=-F_{a i} \cdot \mu
$$

The resulting cut force is given by:

$$
F_{c}=F_{c i} \cdot N_{m}
$$

\section{Kinematic relations}

The mean cutting speed for a forth and back of the blade (i.e. A) is: 


$$
S_{c}=2 \mathrm{Af}
$$

The instantaneous cutting speed has a sinusoidal form:

$$
S_{c}(\mathrm{t})=2 \mathrm{Af} \sin (2 \pi \mathrm{ft})
$$

It can be inferred that the maximal cutting speed is:

$$
S_{c \max }=2 \pi \mathrm{Af}
$$

The instantaneous advance speed during the descending phase is null:

$$
t \in\left[\frac{n}{f}, \frac{1+2 n}{2 f}\right], n \in N \Rightarrow S_{a}(t)=0
$$

The instantaneous advance speed during the cutting - ascending - phase is (see similar triangles on Figure 11):

$$
t \in\left[\frac{1+2 n}{2 f}, \frac{n+1}{f}\right], n \in \mathbb{N} \Rightarrow S_{a}(t)=-\frac{H_{d} S_{c}(t)}{s}
$$

The mean advance speed during one time period is:

During

$$
t \in\left[\frac{n}{f}, \frac{1+2 n}{2 f}\right]
$$

we get

$$
S_{a}=\frac{2 H_{d} f A}{s}
$$

\section{Power relations}

During the cutting phase, the power provided by the engine and the hand must be enough to cut the wood, then:

$$
P_{m}+\left(F_{t}-F_{f}-F_{a}\right) \cdot S_{a}(t) \geq F_{c}(t) S_{c}(t)
$$

Given that $F_{t}-F_{f}-F_{a}=0$ (advance acceleration neglected), then we obtain:

$$
P_{m} \geq F_{c}(t) S_{c}(t)=F_{c} S_{c}(t)
$$

And consequently,

$$
P_{m} \geq F_{c} \max \left(S_{c}(t)\right)
$$

leading to:

$$
P_{m} \geq F_{c} \cdot 2 \pi A f
$$




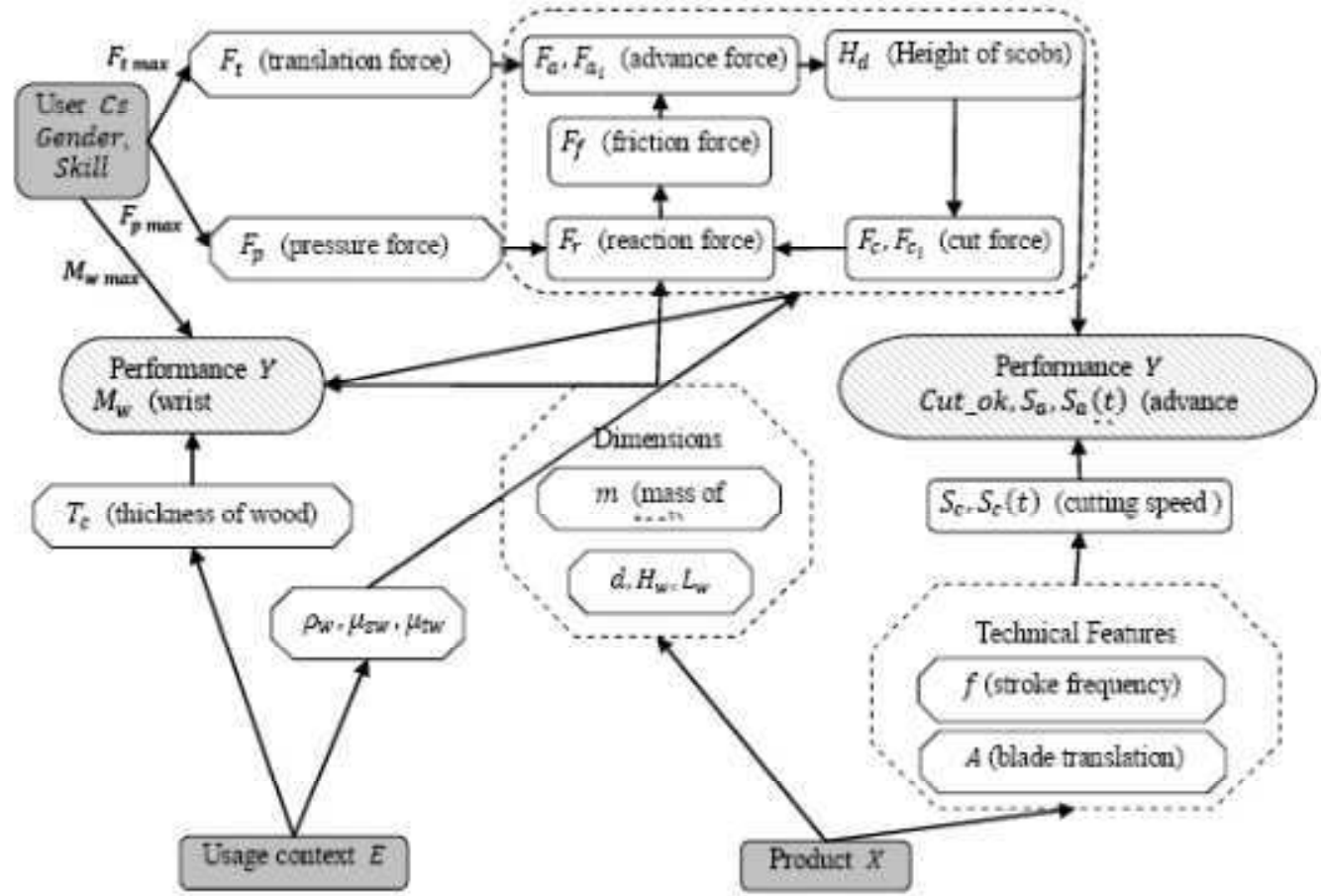

Fig. 10 Causal relations between the variables of the usage coverage modeling issue of a jigsaw 